\title{
Análisis de resultados de futuros profesores de matemática en los contenidos estadísticos y probabilísticos de la evaluación nacional diagnóstica
}

\author{
Francisco Rodríguez-Alveal \\ frodriguez@ubiobio.cl \\ https://orcid.org/0000-0002-9141-4502 \\ Universidad del Bío-Bio (UBB) \\ Chillán, Chile. \\ Danilo Díaz-Levicoy \\ dddiaz01@hotmail.com \\ https://orcid.org/0000-0001-8371-7899 \\ Universidad Católica del Maule (UCM) \\ Talca, Chile.
}

Recibido: 13/09/2020 Aceptado: 12/02/2021

\begin{abstract}
Resumen
El objetivo de este estudio fue analizar los resultados obtenidos por estudiantes de Pedagogía en Educación Matemática, de una universidad chilena en las pruebas de estadística y probabilidad de la Evaluación Nacional Diagnóstica, y su relación con el rendimiento académico en asignaturas disciplinares y pedagógicas de su itinerario formativo. La muestra fue intencional, y estuvo formada por 32 futuros profesores del octavo semestre de la carrera, que rindieron la Evaluación Nacional Diagnóstica en el año 2018. Se analizan los resultados de la prueba de conocimientos pedagógicos y los relacionados con estadística y probabilidad de la prueba de conocimientos disciplinares y didácticos, que fueron proporcionados por el Ministerio de Educación. El análisis se complementa con el rendimiento académico en las asignaturas relacionadas con estadística y con currículo y evaluación de su itinerario formativo. Además, se realizó una entrevista semiestructurada a 13 profesores en formación. Los resultados evidencian que los porcentajes de logro en probabilidad y estadística se relacionan con los correspondientes en Inferencia Estadística, Currículo y Evaluación Educacional I y II. Se concluye la necesidad de conectar las asignaturas de contenidos disciplinarios y pedagógicos/didácticos, como una forma de contribuir a una formación integrada de los futuros profesores.
\end{abstract}

Palabras clave: Formación de profesores. Estadística. Educación Media. Evaluación Nacional Diagnostica.

\section{Análise dos resultados dos futuros professores de matemática nos conteúdos estatísticos e probabilísticos da avaliação diagnóstica nacional}

\section{Resumo}

O objetivo deste estudo foi analisar os resultados obtidos por alunos de Pedagogia em Educação Matemática, de uma universidade chilena, nos testes estatísticos e probabilísticos da Avaliação Diagnóstica Nacional, e sua relação com o desempenho escolar nas disciplinas disciplinares e pedagógicas de seus itinerário de treinamento. A amostra foi intencional e foi composta por 32 futuros professores do oitavo semestre da carreira, que realizaram a Avaliação Diagnóstica Nacional em 2018. São analisados os resultados da prova de conhecimentos pedagógicos e os relacionados. com estatísticas e probabilidade da prova de conhecimentos disciplinares e 
didácticos, disponibilizados pelo Ministério da Educação. A análise é complementada com o desempenho escolar nas disciplinas relacionadas com a estatística e com o currículo e avaliação do seu itinerário formativo. Além disso, foi realizada uma entrevista semiestruturada com treze professores em formação. Os resultados mostram que as percentagens de aproveitamento em probabilidade e estatística estão relacionadas com as correspondentes em Inferência Estatística, Currículo e Avaliação Educacional I e II. Conclui-se a necessidade de articular as disciplinas dos conteúdos disciplinares e pedagógicos / didáticos, como forma de contribuir para uma formação integrada dos futuros professores.

Palavras chave: Formação de professores. Estatística. Educação média. Avaliação Nacional Diagnóstica.

\title{
Analysis of the results of future mathematics teachers in the statistical and probabilistic contents of the national diagnostic assessment
}

\begin{abstract}
The objective of this study was to analyse the results obtained by students of Pedagogy in Mathematics Education, from a Chilean university in the statistical and probability content of the National Diagnostic Assessment, and their relationship with their academic performance in disciplinary and pedagogical subjects of their training itinerary. The sample was intentional, and consisted of 32 prospective teachers from the eighth semester of the career, who took the National Diagnostic Assessment in 2018. The results of the pedagogical knowledge test and those related. with statistics and probability of the disciplinary and didactic knowledge test, which were provided by the Ministry of Education are analysed. The analysis is complemented with the academic performance in the subjects related to statistics and with the curriculum and evaluation of their training itinerary. In addition, a semi-structured interview was conducted with 13 prospective teachers. The results show that the achievement percentages in probability and statistics are related to the corresponding scores in Statistical Inference, Curriculum and Educational Evaluation I and II. We conclude the need to connect the subjects of disciplinary and pedagogical / didactic content, as a way of contributing to an integrated training of prospective teachers.
\end{abstract}

Keywords: Teacher training. Statistic. Middle education. National Diagnostic Assessment.

\section{Introducción}

Desde la inserción a partir del año 2009 de los tópicos acerca de la estadística y probabilidad en el currículo escolar chileno de educación media (en el eje de datos y azar,) establecido por el Ministerio de Educación (MINEDUC, 2009), se ha mostrado que es una temática fundamental para el desarrollo del pensamiento crítico y la formación de una cultura estadística, y para la formación ciudadana (Engel, 2019; Gal, 2002, 2004, 2019).

Ello es producto de la gran cantidad de información resumida de manera gráfica y numérica con la cual interactuamos diariamente en los medios masivos de comunicación, y de la divulgación de estadísticas oficiales y los reportes de investigación en numerosos campos del 
saber (Arteaga, Batanero, Cañadas, \& Contreras, 2011). La comprensión de dicha información requiere que los profesores den prioridad a las habilidades de leer, interpretar, evaluar críticamente y apreciar la información estadística en el contexto en que se está inmersa (BenZvi \& Garfield, 2004; Engel, 2019; Gal, 2002; Zapata-Cardona, 2016).

Estas temáticas han tensionado los itinerarios de las instituciones formadoras de profesores de matemática, quienes deben familiarizarse con su objeto de estudio (Cobb \& Moore, 1997). Este escenario ha abierto a nivel nacional un debate acerca de la calidad de los procesos de enseñanza, aprendizaje y evaluación de estos contenidos, tanto a nivel escolar como universitario (Estrella, Olfos \& Mena-Lorca, 2015), debido a los bajos resultados que han obtenido los futuros profesores en pruebas estandarizadas.

Una consecuencia, de este debate es que las instituciones de educación superior han reformulado sus mallas curriculares y programas de asignaturas, y adecuado sus perfiles de egreso a las nuevas demandas ministeriales, situación que actualmente están llevando a cabo la mayoría de las carreras de pedagogía de Chile. Con lo cual, se espera revertir el comentario realizado por el MINEDUC, acerca que, a pesar que la estadística y probabilidad están insertas en el currículo escolar hace alrededor de una década, "no existe una cultura de su enseñanza" (MINEDUC, 2012, p. 94) y, por consiguiente, son escasos los procesos evaluativos tendientes a generar aprendizajes significativos en los estudiantes.

En este contexto, el MINEDUC el año 2016 introduce la Ley 20.903, uno de cuyos puntos establece que los estudiantes de pedagogía, al inicio de su formación y antes de titularse, deben rendir una prueba denominada Evaluación Nacional Diagnóstica (en adelante END), con el objetivo de comprobar la formación inicial lograda en las instituciones de educación superior. En particular, hay preocupación por los bajos resultados que obtienen los estudiantes del sistema escolar en las evaluaciones estandarizadas, como el Sistema de Medición de la Calidad de la Educación (SIMCE), que rinden los estudiantes durante su Educación Básica y Media, y la Prueba de Selección Universitaria (PSU), previa al ingreso a la educación superior en Chile, puntualmente en temáticas asociadas a estadística y probabilidad. Estos resultados generan un desafío para las instituciones formadoras y para quienes se están preparando como futuros profesores del sistema escolar, ya que deben tratar de dar respuesta a los requerimientos de una sociedad que cambia constantemente (Vaillant \& Marcelo, 2001).

Producto de lo anterior, resulta de interés indagar acerca de las debilidades y fortalezas 
que presentan los futuros profesores próximos a egresar en torno a los conocimientos disciplinarios y pedagógicos y particularmente como se relacionan con el rendimiento académico. Es por ello, que en este trabajo nos planteamos los siguientes objetivos:

- Evaluar los resultados obtenidos por los estudiantes de Pedagogía en Educación Matemática de una universidad chilena en la END en las temáticas de estadística y probabilidades.

- Correlacionar los resultados obtenidos por los participantes en la END con el rendimiento académico de las asignaturas disciplinares afines a estadística y probabilidad, y las pedagógicas asociadas a la evaluación cursadas durante su itinerario formativo.

\section{Contexto del estudio}

La Formación Inicial Docente (FID), según Marcelo y Vailllant (2018a), es un escenario complejo en el que intervienen diferentes aspectos, como tradiciones, tendencias profesionales, instituciones y procesos formativos. Chile no ha estado ajeno de esta realidad, dado que existe una amplia oferta de carreras pedagógicas, que según Avalos (2008) es "fragmentada y carente de regulación externa y sobre la que existen dudas respecto a la capacidad docente de muchos de sus egresados" (p. 276). En este sentido, Vezub (2016) explicita que "existe un diagnóstico extendido que alerta sobre la falta de calidad y pertinencia de la formación inicial y la necesidad de acercarla a las demandas reales de la profesión" (p. 2).

En consecuencia, el MINEDUC ha formulado políticas tendientes a potenciar la FID, aunque estas iniciativas han sido cuestionadas, dado que no han tenido los impactos que se esperaba. La iniciativa más reciente es la promulgación y puesta en marcha Ley 20.903 de Desarrollo Profesional Docente (MINEDUC, 2016), cuyos puntos centrales son los mecanismos de evaluación y los procesos de enseñanza y aprendizaje realizados por las instituciones formadoras, mediante la END a las que deben someterse los estudiantes de pedagogía como requisito de titulación. Dicha prueba se subdivide en una Prueba de Conocimientos Pedagógicos Generales (PCPG) y otra de Conocimientos Disciplinarios y Didácticos (PCDD), que se encuentran alineadas con los Estándares Orientadores para las carreras de Pedagogía en Educación Media (MINEDUC, 2012). También entrega directrices para regular la calidad de la formación de los egresados de las carreras de pedagogía en áreas como lenguaje y 
comunicación, matemática, historia, geografía y ciencias sociales, biología, física y química.

La prueba de conocimientos pedagógicos generales (PCPG) se compone de 50 reactivos de selección múltiple, que, para los estudiantes de Pedagogía en Educación Media, se distribuyen en torno a tres ejes temáticos: aprendizaje y desarrollo de los estudiantes de educación media (19 ítems); diseño e implementación de la enseñanza (21 ítems); y la profesión docente y el sistema educacional chileno (10 ítems). Por otro lado, la prueba PCDD, que deben rendir los estudiantes de pedagogía en matemática es una prueba de selección múltiple, que consta de 60 ítems a través de los que se evalúan los contenidos asociados a sistemas numéricos y álgebra (24 ítems); cálculo (4 ítems); estructuras algebraicas (4 ítems); geometría (15 ítems) y estadística y probabilidad (13 ítems). Hacemos notar que los tópicos afines a estadística y probabilidad del eje de datos y azar son relativamente nuevos en el currículo escolar para la educación media, pues han sido introducidos en el marco curricular por el MINEDUC el año 2009. Asimismo, este eje temático tiene asociados "cinco estándares que describen los conocimientos, habilidades y competencias que el futuro profesor o profesora necesita para conducir con éxito el proceso de enseñanza y aprendizaje de los estudiantes de enseñanza media” (MINEDUC, 2012, p. 92). De los cuales, la PCDD evalúa los siguientes estándares (MINEDUC, 2012, p. 21):

Estándar 17: Es capaz de motivar la recolección y estudio de datos y de conducir el aprendizaje de las herramientas básicas de su representación y análisis.

Estándar 18: Es capaz de conducir el aprendizaje de las probabilidades discretas.

Estándar 20: Está preparado para conducir el aprendizaje de la distribución normal y teoremas límite.

Estándar 21: Está preparado para conducir el aprendizaje de inferencia estadística.

Estos estándares dan cuenta de tópicos afines con estadística descriptiva, probabilidad, distribuciones de probabilidad discretas y continuas, como la binomial y normal, e inferencia estadística. Los saberes disciplinarios y pedagógicos adquiridos por los estudiantes de pedagogía en matemática juegan un rol fundamental en su formación, dado que ellos serán los encargados de comunicar estos conocimientos a los estudiantes del sistema escolar, futuros ciudadanos de un mundo cada día más globalizado.

En este contexto, se interpela a las instituciones y a los docentes universitarios 
formadores de formadores, para que estos futuros profesores adquieran las habilidades $\mathrm{y}$ conocimientos asociados a los tópicos de estadística y probabilidad, atendiendo a los estudios a nivel nacional que evidencian bajos niveles en comprensión de información resumida en representaciones gráficas presentes en el currículo escolar chileno por profesores en formación y en activo (Rodríguez-Alveal \& Sandoval, 2012).

\section{Fundamentos}

\section{El conocimiento especializado del profesor de matemática}

Desde hace más de una década, investigadores como Ponte y Chapman (2006) han puesto su atención sobre la formación, el conocimiento y el desarrollo profesional del profesor de matemática. En este contexto, Shulman (1986) fue uno de los pioneros a nivel mundial en realizar estudios en relación al conocimiento del profesor para la enseñanza; proponiendo un modelo con tres componentes del conocimiento del profesor: un conocimiento del contenido (Content Knowledge, CK), un conocimiento pedagógico (Pedagogical Knowledge, PK) y un conocimiento pedagógico del contenido o conocimiento didáctico del contenido (Pedagogical Content knowledge, PCK), el cual es producto de la interacción entre el conocimiento del contenido y el conocimiento pedagógico.

En este y otros modelos el supuesto es que no basta para el profesor con dominar el conocimiento conceptual de la materia, sino que también es necesario el componente

pedagógico. Aunque la propuesta de Shulman (1986) aun continúa vigente, Ball, Thames y Phelps (2008) adaptan su modelo e introducen subdominios en el conocimiento de los contenidos matemáticos (común, especializado y en el horizonte). Al respecto, Hill, Ball y Schilling (2008) mencionan que el "conocimiento común del contenido, es el que posee cualquier persona instruida en matemática; el conocimiento especializado del contenido, aplicado por el profesor para articular tareas de enseñanza acerca de tópicos por ejemplo relacionados con estadística y probabilidad”.

\section{Algunos antecedentes}

Aunque la investigación sobre formación de profesores para enseñar estadística fue escasa hasta hace una década, comienza a desarrollarse a partir del ICMI estudios sobre el tema (Batanero, Burrill, \& Reading, 2011). A continuación, describimos algunas investigaciones 
actuales.

En relación al conocimiento de los gráficos estadísticos, Batanero, Arteaga y Ruiz (2010) en un estudio realizado en futuros profesores de educación primaria, muestran que solamente una parte de los participantes logran construir gráficos con la complejidad semiótica necesaria para obtener conclusiones, presentando además un nivel de elemental al interpretar la información de los gráficos. Coutinho, Almouloud y Silva (2012), por su parte realizaron un estudio sobre la construcción e interpretación de gráficas estadísticas por profesores de matemática haciendo uso del software Geogebra. Entre los hallazgos, se destacan que los profesores participantes no logran un adecuado desempeño en el uso del software y la construcción de representaciones gráficas de manera autónoma, evidenciándose bajas habilidades en la interpretación de información resumida en dichas representaciones. Estas carencias se confirman en un estudio de Gea, Arteaga y Cañadas (2017) con futuros profesores de educación secundaria, que tuvieron dificultad en interpretar un diagrama acumulativo de frecuencias y un gráfico de caja.

Por oro lado, son varios los trabajos sobre comprensión de las medidas de tendencia central. Entre ellos, Gea, Batanero, Fernandes, y Arteaga (2016) describen las dificultades de futuros profesores de educación secundaria para interpretar los percentiles, y elegir una medida de tendencia central adecuada a un conjunto de datos. Rodríguez-Alveal, Maldonado-Fuentes y Sandoval (2016) develan que los profesores en formación de pedagogía en matemática presentan porcentajes de logro menores en relación al cálculo de resúmenes estadísticos, tales como media aritmética y la media ponderada, en presencia de información explicita con datos agrupados (tablas de distribución de frecuencia). Además, en relación con la evaluación de conocimientos y capacidad argumentativa adquiridos por los profesores de matemática, Rodríguez-Alveal, Díaz-Levicoy y Maldonado-Fuentes (2018) observan que dichos profesores no presentan dificultades procedimentales para el cálculo de la media aritmética, mediana y moda. No obstante, los argumentos estadísticos utilizados para justificar la elección de estos índices en la toma de decisiones son poco adecuados.

Estos resultados se repiten en los mismos grupos de estudio en temas asociados a la probabilidad, ante situaciones problemáticas donde se encuentra presente la aleatoriedad, empleando argumentaciones poco pertinentes para explicar por qué una sucesión de eventos es aleatoria o no (Rodríguez-Alveal, Díaz-Levicoy, \& Vásquez, 2018), a pesar de que este 
contenido se encuentra presentes en el currículo escolar y en los libros distribuidos por el Ministerio de Educación a los estudiantes de enseñanza básica y media de establecimientos educacionales municipalizados y subvencionados por el Estado en Chile. Por consecuencia, estos resultados evidencian que los estudiantes en formación de Pedagogía en Matemática no han consiguen una alfabetización numérica ni probabilística, durante su proceso formativo.

Respecto al tema de correlación y regresión, Gea, Batanero, Arteaga. y Estepa (2019) investigan los conocimientos común, avanzado y especializado del tema en futuros profesores de bachillerato. Aunque los participantes tienen un buen conocimiento matemático del tema, muestran dificultades para elegir una recta de ajuste a unos datos y para explicar las razones por las que un conjunto de datos presenta correlación. En otro estudio Fernandes, Gea y Ferreira (2019) evalúan el conocimiento estadístico común de un grupo de futuros profesores de educación primaria cuando resuelven una tarea que involucra una distribución bidimensional, con relación a su gráfico, correlación lineal y regresión lineal. Entre los resultados destacan que los futuros profesores tienen mayor éxito al realizar la gráfica de la distribución y presentan mayores dificultades al trabajar con la regresión lineal.

En relación con el PK o conocimiento didáctico del contenido, Gómez et al. (2014) muestra que los profesores en formación presentan una pobre intuiciones acerca de fenómenos aleatorios, cuyos resultados coinciden con el estudio de Rodríguez-Alveal, Díaz-Levicoy, Vásquez (2018). Además, el estudio de Gómez et al. (2014) muestra que los participantes presentan una escasa capacidad para identificar los objetos matemáticos presentes en una tarea, incluso cuando los futuros profesores utilizaron dichos objetos al resolver correctamente los problemas planteados. Asimismo, los profesores en formación al analizar las respuestas entregadas por cuatro alumnos, fueron capaces de discriminar las respuestas correctas e incorrectas. En resumen, es de interés ahondar en el componente didáctico en esta área lo cual sería de gran ayuda en su desempeño en el sistema escolar.

Estos antecedentes confirman lo expuesto por Marcelo y Vaillant (2018b), respecto a que los programas de formación para docentes "no están dando los resultados esperados tanto en lo pedagógico como en lo disciplinar debido, entre otras causas, a que los programas de asignaturas presentan arquitecturas que propician el aislamiento" (p. 13), situación que no permite la necesaria articulación para aprender a enseñar, lo que repercutiría en los instrumentos y prácticas evaluativas. 


\section{Método}

\section{Diseño del estudio}

El presente estudio es de carácter exploratorio, al considerar una muestra de tamaño limitado. En su abordaje se considera complementariedad metodológica, utilizando técnicas mixtas en la recolección de datos, lo que permite que un método compense la debilidad del otro, facilitando la interpretación de los datos y aumentando la confianza y validez de los resultados (Cornejo, Sanhueza, \& Rioseco, 2012).

Desde el punto de vista cuantitativo, se compararon los porcentajes de logro promedio de los estudiantes en la END del año 2018, en la prueba de conocimientos disciplinares y didácticos, con su rendimiento académico en las asignaturas relacionadas con estadística, probabilidad y evaluación, tendiente a explicar dichos resultados. Mientras que la parte cualitativa fue de profundización y consistió en entrevistas individuales a una submuestra de participantes a los cuales se les interrogó acerca de su formación en las asignaturas de estadística, probabilidad y evaluación.

\section{Contexto y participantes}

La muestra participante en la investigación está formada por estudiantes de la carrera de Pedagogía en Educación Matemática de una universidad ubicada geográficamente en el centrosur de Chile. Esta institución cuenta con una trayectoria de más de cuarenta años de experiencia en la formación de profesores y forma parte del Consejo de Rectores de Universidades chilenas (CRUCH). Los participantes del presente estudio fueron seleccionados mediante un muestreo no probabilístico, del tipo estratégico o intencional (McMillan \& Schumacher, 2011). Se tuvieron en cuenta los siguientes criterios de inclusión: estudiantes de octavo semestre de la carrera de Pedagogía en Educación Matemática y que rindieron la END en el año 2018 de acuerdo con lo establecido en la Ley 20.903. En base a estos criterios el número de participantes fue de 32 , donde el 57,6\% eran hombres y el 42,4\% mujeres, de los cuales, 13 aceptaron participar de manera voluntaria de una entrevista semiestructura.

\section{Instrumentos de recolección de datos}

En este estudio utilizamos una base de datos proporcionada por el MINEDUC, atendiendo a la Ley de transparencia (Ley 20.285), en la cual se entregan los resultados 
obtenidos por los estudiantes de Pedagogía en Educación Matemática que participaron de la END el año 2018 en las pruebas de conocimientos pedagógicos, en la cual se evaluaron los siguientes contenidos:

a. Diseño e implementación de la enseñanza. Conocimiento del currículo nacional vigente, su propósito general y sus principios inspiradores, su estructura y secuencia.

b. La profesión docente y el sistema educacional chileno. Toma de decisiones pedagógicas a partir del análisis y reflexión individual y colectiva sobre la propia práctica pedagógica y sobre los resultados de aprendizaje de los estudiantes.

De manera similar, se utilizó información asociada a los contenidos de estadística y probabilidad de la prueba de conocimientos disciplinares y didácticos de la END. Dicha información fue complementada con el rendimiento académico (calificaciones) de los estudiantes en las asignaturas relacionadas con estadística y probabilidad. Las cuales tienen por objetivo que los estudiantes sean capaces de reconocer y aplicar algunos métodos y técnicas básicas de la estadística descriptiva, probabilidad e inferencia estadística, tales como estimación de parámetros y pruebas de hipótesis, temáticas que son abordadas en la END.

Por otro lado, las asignaturas relacionadas con evaluación tienen como propósito que los estudiantes adquieran una visión global acerca del currículo, didáctica y evaluación vinculando los procesos de planificación curricular con el diseño de la enseñanza y con la evaluación. Estas asignaturas son transversales a las ocho carreras de pedagogía adscritas a la Facultad de Educación de la institución formadora. incluida la rama de Educación Matemática, a la cual pertenecen los participantes de esta investigación.

El segundo instrumento, consistió en una entrevista semiestructurada a trece profesores en formación, la que buscó recolectar información acerca de la incidencia de la formación en estadística, probabilidad y evaluación en los resultados obtenidos en la END. Al respecto, se consideraron las siguientes preguntas:

- ¿Cree usted que la interacción entre los cursos de estadística, probabilidad y evaluación presentes en el itinerario formativo permitirían dar respuesta a las habilidades que demanda la estadística y probabilidad en el sistema escolar?

- ¿Qué contenidos recuerda usted de las asignaturas de estadística y probabilidad realizados durante su formación inicial? 


\section{Análisis de la información}

Para el análisis de la información cuantitativa se consideraron métodos de estadística descriptiva univariada numérica (porcentajes, promedios), coeficiente de asimetría, y coeficiente de correlación de Pearson. El procesamiento de la información cuantitativa fue realizado en el paquete estadístico SPSS 13.0 y XLSTAT.

En la segunda fase se transcribieron y analizaron los discursos grabados en formato de audio en forma literal entregados por los 13 profesores en formación entrevistados. Este número de participantes se encuentra dentro del rango $15 \pm 10$ mencionado como adecuado por Kvale (2011). En el análisis de los relatos entregados por los participantes se hizo uso de los siguientes pasos del modelo propuesto por Mayring (2000) para el análisis de datos cualitativos:

- Pre-análisis. Se consideraron las transcripciones literales de las grabaciones de audio de las entrevistas realizadas a los estudiantes en FID acerca de las preguntas afines a las temáticas de estadística y probabilidad y evaluación.

- Definición de las unidades de análisis. Se seleccionaron segmentos de los relatos entregados por los participantes acerca de la formación recibida en las asignaturas del área disciplinar afines a estadística y probabilidad y de las asignaturas pedagógicas, relacionadas con evaluación y al objetivo del estudio.

- Desarrollo de categorías. Se analizó el corpus de las entrevistas realizadas a los participantes identificando categorías afines al objetivo de la investigación, para formarse una idea acerca de las similitudes o discrepancias de las respuestas entregadas, de manera de ampliar las posibilidades interpretativas.

- Integración de hallazgos. Corresponde a la fase final de la metodología del análisis de contenido de las entrevistas que rescata las percepciones de los participantes en relación a las interrogantes planteadas en la entrevista.

\section{Resultados}

En la presente sección se presentan los resultados, siguiendo la estructura de los objetivos previamente descritos. 


\section{Rendimiento académico de los participantes en estadística y evaluación}

En la Tabla 1 se muestran los promedios, desviación estándar y coeficiente de asimetría del rendimiento académico de los 32 estudiantes de Pedagogía en Educación Matemática que rindieron la END el año 2018.

Tabla 1

Resúmenes estadísticos del rendimiento académico en las asignaturas relacionadas con estadística, probabilidad y evaluación de los participantes en el estudio

\begin{tabular}{lccc}
\hline \multicolumn{1}{c}{ Asignaturas } & Media & $\begin{array}{c}\text { Desviación } \\
\text { típica }\end{array}$ & $\begin{array}{c}\text { Coeficiente de } \\
\text { asimetría }\end{array}$ \\
\hline Métodos Estadísticos y Probabilidad & 4,6 & 0,5 & 0,9 \\
Inferencia Estadística & 4,4 & 0,8 & $-0,5$ \\
Didáctica de la Estadística & 4,3 & 0,5 & $-0,2$ \\
Currículo y Evaluación Educacional I & 4,5 & 0,4 & 0,0 \\
Currículo y Evaluación Educacional II & 4,6 & 0,4 & 0,5 \\
\hline
\end{tabular}

Fuente: elaborado por los autores

En dicha tabla se observa que, en general, el promedio obtenido por los estudiantes, tanto en las asignaturas de especialidad relacionadas con estadística y pedagógicas afines a evaluación, son relativamente similares, del orden del 4,3 al 4,6 (en una escala de 1,0 al 7,0) Situación, similar ocurre con la variabilidad de sus calificaciones, aunque en Inferencia Estadística la mayor variabilidad entre las calificaciones de los estudiantes evidencia la complejidad de los contenidos como las distribuciones muestrales del promedio y la proporción, su relación con estimaciones puntales y probabilística y la toma de decisiones en base a pruebas de hipótesis. Igualmente muestra la desigual a comprensión de conceptos estadísticos y probabilísticos como estimadores, insesgadez, parámetros y errores de muestreo.

Por otra parte, se observó que la distribución de las calificaciones de los futuros profesores presentó una distribución asimétrica negativa, en el caso de las asignaturas de Inferencia Estadística y Didáctica de la Estadística, evidenciando que las puntuaciones tienden a concentrarse en cifras por debajo del promedio. En cambio, en las asignaturas de Métodos Estadísticos y probabilidad, y Currículo y Evaluación Educacional II presentan asimetrías positivas, indicando que en esta asignatura los estudiantes obtienen más calificaciones por encima del promedio. Solamente en la asignatura de Currículo y Evaluación Educacional I las 
calificaciones tienden a distribuirse de manera relativamente simétrica.

Asimismo, se observa que en general los resultados promedios de los estudiantes son relativamente homogéneos salvo en la asignatura de inferencia estadística, lo anterior se podría explicar en que dichas asignaturas son frecuentes el uso de trabajos grupales como una forma de evaluar la adquisición de los contenidos, lo que tienen una alta ponderación en el promedio de cada estudiante. Todo ello teniendo en cuenta que no hay diferencias en las notas medias, todas ellas por encima de la media teórica (4) aunque muy cerca de ella.

\section{Resultados de los participantes en la Evaluación Nacional Diagnostica}

En la Tabla 2 se muestran los porcentajes de logro obtenidos por los estudiantes de Pedagogía en Educación Matemática en la prueba END.

Tabla 2

Resúmenes estadísticos del porcentaje de logro obtenido por los participantes en la END

\begin{tabular}{lccc}
\hline Pruebas de Conocimientos & Media & $\begin{array}{c}\text { Desviación } \\
\text { típica }\end{array}$ & $\begin{array}{c}\text { Coeficiente } \\
\text { de asimetría }\end{array}$ \\
\hline $\begin{array}{l}\text { Aprendizaje y desarrollo de estudiantes de } \\
\text { Educación Media (PCPG-Tema 1) }\end{array}$ & 53,5 & 14,2 & 0,0 \\
$\begin{array}{l}\text { Diseño e implementación de la enseñanza } \\
\text { (PCPG-Tema 2) }\end{array}$ & 56,3 & 13,0 & $-0,3$ \\
Estadística y Probabilidad & & & \\
\hline
\end{tabular}

Fuente: elaborado por los autores

El porcentaje de logro en la prueba sobre Aprendizaje y desarrollo de estudiantes de Educación Media oscila entre 21,1 y 78,9, en la prueba sobre Diseño e implementación de la enseñanza entre 19 y 85,7 y en la relacionada con los tópicos afines a estadística y probabilidad fluctúa entre 0,0 y 76,9 respectivamente. Se observa que el promedio de porcentaje de logro más alto obtenido por los estudiantes fue el relacionado con el diseño e implementación de la enseñanza (PCPG-Tema 2), cuya distribución es simetría, seguido por el aprendizaje y desarrollo de estudiantes de educación media (PCPG-Tema1) de la prueba de conocimientos pedagógicos generales con una asimetría negativa. Por tanto, se muestra un mejor conocimiento del contenido y la enseñanza que del contenido y el estudiante (en la terminología de Ball, Thames y Phelps, 2008).

No obstante, dentro los temas relacionados con la prueba de conocimientos disciplinarios 
y didácticos en estadística y probabilidad, los estudiantes obtienen en promedio un porcentaje de logro más bajo, con asimetría negativa cuyos resultados en general se concentran en porcentajes de logros bajos, lo que evidencia un rendimiento menor en el conocimiento común de los contenidos asociados a estadística y probabilidad. Estos, resultados que se condicen con el rendimiento académico obtenido en estas temáticas por los estudiantes de Pedagogía en Educación Matemática presentes en este estudio (Tabla 1).

Tabla 3

Matriz de correlaciones entre porcentajes de logro en la END y el rendimiento académico de los estudiantes en las asignaturas afines con estadística, probabilidad y evaluación.

\begin{tabular}{|c|c|c|c|c|c|c|c|}
\hline & \multicolumn{3}{|c|}{ Resultados END } & \multicolumn{4}{|c|}{ Asignaturas en su formación } \\
\hline & 1 & 2 & 3 & 4 & 5 & 6 & 7 \\
\hline \multicolumn{8}{|l|}{ Resultados en la END } \\
\hline 1. PCPG-Tema 1 & -- & & & & & & \\
\hline 2. PCPG-Tema 2 & $0,41 *$ & -- & & & & & \\
\hline$p$ (bilateral) & 0,02 & & & & & & \\
\hline $\begin{array}{l}\text { 3. Estadística y } \\
\text { Probabilidad }\end{array}$ & $0,36^{*}$ & $0,59 * *$ & -- & & & & \\
\hline$p($ bilateral $)$ & 0,04 & 0,00 & & & & & \\
\hline \multicolumn{8}{|l|}{ Asignaturas en su formación } \\
\hline $\begin{array}{l}\text { 4. Métodos Estadísticos y } \\
\text { Probabilidad }\end{array}$ & $0,39 *$ & 0,17 & 0,22 & -- & & & \\
\hline$p($ bilateral $)$ & 0,04 & 0,38 & 0,26 & & & & \\
\hline $\begin{array}{l}\text { 5. Inferencia Estadística } \\
\qquad p(\text { bilateral) }\end{array}$ & $\begin{array}{l}0,20 \\
0,33\end{array}$ & $\begin{array}{l}0,34 \\
0,09\end{array}$ & $\begin{array}{c}0,44^{*} \\
0,03\end{array}$ & $\begin{array}{l}0,19 \\
0,37\end{array}$ & -- & & \\
\hline $\begin{array}{l}\text { 6. Didáctica de la } \\
\text { Estadística }\end{array}$ & 0,36 & 0,40 & $0,47 *$ & 0,17 & $0,69 * *$ & -- & \\
\hline$p($ bilateral $)$ & 0,08 & 0,05 & 0,02 & 0,43 & 0,00 & & \\
\hline $\begin{array}{l}\text { 7. Currículo y Evaluación } \\
\text { Educacional I }\end{array}$ & $0,46^{*}$ & $0,58 * *$ & $0,49 * *$ & 0,29 & 0,36 & $0,64 * *$ & -- \\
\hline$p($ bilateral $)$ & 0,01 & 0,00 & 0,01 & 0,14 & 0,14 & 0,00 & \\
\hline $\begin{array}{l}\text { 8. Currículo y Evaluación } \\
\text { Educacional II }\end{array}$ & $0,41 *$ & $0,52 * *$ & $0,44^{*}$ & 0,38 & $0,40^{*}$ & $0,47^{*}$ & $0,55 * *$ \\
\hline$p($ bilateral $)$ & 0,03 & 0,00 & 0,02 & 0,05 & 0,04 & 0,02 & 0,00 \\
\hline
\end{tabular}

Fuente: elaborado por los autores 
Para estudiar la relación de puntuaciones en las pruebas consideradas en END y el rendimiento académico de los estudiantes en las asignaturas de su formación afines con estadística, probabilidad y evaluación en la Tabla 3 se muestra, la correlación entre los porcentajes de logro obtenidos en los componentes analizados en las secciones anteriores.

Se observa que existe una correlación directa estadísticamente significativa entre los porcentajes de logro promedio de los resultados END en Diseño e Implementación del Currículo-Tema 2 y las temáticas relacionadas con estadística y probabilidad de la END Currículo y Evaluación Educacional I), y las asignaturas de su formación Currículo y Evaluación Educacional II. Dichas asignaturas según sus programas tienen como propósito entregar un enfoque teórico-práctico de manera que los estudiantes vinculen el currículo, la didáctica y la evaluación con el proceso enseñanza-aprendizaje a nivel de sistema escolar.

Respecto a los resultados obtenidos en la END por los estudiantes en tópicos relacionados con estadística y probabilidad se correlacionan de manera significativa con los resultados de su formación en Inferencia Estadística, Didáctica de la Estadística Currículo y Evaluación Educacional I y Currículo y Evaluación Educacional II.

En resumen, las correlaciones evidencian que el rendimiento académico promedio de los estudiantes en las asignaturas relacionadas con estadística y probabilidad, de currículo y evaluación tiene relación directa con el logro en las pruebas analizadas de la END. Ello se explica porque las asignaturas afines a evaluación tienen por objetivo entregar antecedentes generales acerca del currículo, la didáctica y la evaluación, sin abordar las especificidades y particularidades de cada una de las carreras de pedagogía, en particular la de pedagogía en educación matemática. Igualmente, en la Tabla 3 se observa que algunas asignaturas de la formación se correlacionen entre sí. (Inferencia y didáctica de la estadística, ésta con currículo y evaluación educacional I y las dos asignaturas sobre currículo y evaluación.

De las asignaturas de estadística y probabilidad presentes en el itinerario formativo de los estudiantes de pedagogía en matemática, futuros profesores, solamente la asignatura de Métodos Estadísticos y probabilidad no se correlaciona de manera significativa con los porcentajes de logro de las pruebas de la END y con el rendimiento académico de las asignaturas de Currículo y Evaluación Educacional I y II, respectivamente. Lo que estaría evidencia un sesgo en la construcción de la END acerca de las preguntas afines a tópico presentes en la asignatura de Métodos Estadísticos y probabilidad tales como estadística descriptiva, probabilidad y 
regresión lineal, los que tiene una gran presencia en el currículo escolar chileno desde la enseñanza básica. Como así también los temas disciplinares tratados en las asignaturas afines a evaluación.

\section{Reflexiones acerca de la formación disciplinar y pedagógica, y su relación con los resultados en la END}

A continuación, se presentan los resultados del análisis de las entrevistas haciendo uso de las reflexiones de los 13 profesores participantes. De ellos, 8 comentan que durante su formación no existió un dialogo o articulación entre los contenidos disciplinares asociados a estadística y los pedagógicos relacionados con currículo y evaluación. Esta carencia también ha sido reportada en la literatura internacional, por investigadores como Marcelo (2009) y Vaillant (2007). El primero hace mención que la formación disciplinar no interactúa con la pedagógica en la formación de profesores, fenómeno que Feiman-Nemser y Buchman (1988) ha denominado la laguna de dos mundos. Estos resultados estarían evidenciando que los programas de las asignaturas disciplinares y pedagógicas en estudio, así como la articulación entre ellas. presentan problemas estructurales en sus diseños Al respecto, los entrevistados comentan que:

(...) en la carrera no existió una conexión entre las asignaturas de currículo y la evaluación con los contenidos de las asignaturas de estadística y probabilidad (Estudiante 3 ).

(...) una conexión entre estadística y evaluación, ...yo creo que, si tuviese que juntar así tal como yo, que tuve inferencia estadística y métodos, por ejemplo, y currículo (...) no podría sacar nada de eso, porque para mí, sinceramente, métodos e inferencia fueron materias inútiles (Estudiante 5).

Porque (...) yo considero que lo que aprendí en métodos estadísticos y probabilidad o lo poco que aprendí (...) ni siquiera tiene tanta relación con el sistema escolar (...) o no se enseña en la universidad de la forma como aparece en el sistema escolar (Estudiante 8).

Estos comentarios resultan contradictorio, debido que una de las temáticas desarrolladas en la formación inicial, tiene relación con estadística descriptiva; son los contenidos asociados a los Estándares Orientadores Pedagógicos y disciplinarios (MINEDUC, 2012, p. 124), en el cual los futuros profesores deben ser "capaz de motivar la recolección y estudio de datos y de conducir el aprendizaje de las herramientas básicas de su representación y análisis", actividad que está presente desde la básica a la media en el currículo escolar chileno. El porcentaje de logro en este tema alcanza un $35,2 \%$ en el grupo objeto de estudio, porcentaje por debajo del 
promedio a nivel nacional (41,6\%).

Sólo dos de los 13 estudiantes entrevistados, hacen mención a temáticas relacionadas con Estadística descriptiva. Al respecto, uno de los estudiantes explicita algunas medidas de tendencia central y variabilidad, presentes en el currículo escolar, además de algunas pruebas de hipótesis estudiadas en la asignatura de métodos estadísticos y probabilidad e inferencia estadísticas respectivamente:

Vimos la mediana, la media, las desviaciones (...) en la asignatura de métodos estadísticos y probabilidad y algunas pruebas estadísticas que no recuerdo su nombre en la asignatura de inferencia estadística, pero las sé aplicar (Estudiante 1).

En cambio, el otro entrevistado describe temáticas relacionadas con estadística descriptiva, aunque ilustra solo algunos de los tópicos presentes en el programa de asignatura, como las representaciones gráficas, sin indicar que tipos de gráficos estudiaron y el rol desempeñan en estadística descriptiva.

Sí vimos las medidas de tendencia, y las tablas de frecuencia; desarrollamos, los distintos tipos de gráficas, cómo interpretar los datos y cómo analizarlos básicamente (Estudiante 8).

Por el contrario, otra estudiante comenta que, ni en su paso por educación secundaria, ni a nivel universitario le enseñaron estadística descriptiva:

Considerando que no tuve ningún curso de estadística (en enseñanza secundaria), ya que nos saltamos toda la parte de estadística descriptiva. Lo que eran los gráficos, la moda, la mediana nunca lo vimos en la universidad en las asignaturas de estadística y probabilidad (Estudiante 9).

Esta realidad, por lo manifestado por los entrevistado, su formación les ha generado un vacío de conocimiento estadístico; no obstante, la asignatura de didáctica de la estadística le ha permitido adquirirlos, y así también darse cuenta de la importancia de la estadística y probabilidad en el sistema escolar, es decir, ha sido el nexo entre lo disciplinar y lo pedagógico:

Lo comprendí, cuando tuve didáctica de la estadística, asignatura que me permitió darme cuenta de la importancia de la estadística y probabilidad. Pero yo considero que lo que aprendí en las asignaturas de estadística y probabilidad no me va servir mucho para desempeñarme como profesor en el sistema escolar (Estudiante 9). 
Los porcentajes de logro de los estudiantes considerados en el estudio se encuentran por debajo del promedio nacional, siendo el más bajo, en el cual los estudiantes obtienen un 46,9\% de logro en contra de un $60,7 \%$ a nivel nacional, según informe enviado por el Centro de Perfeccionamiento, Experimentación e Investigaciones Pedagógicas (CPEIP). Se trató de indagar en las causas de estos bajos rendimientos. Al respecto dos estudiantes en formación entrevistados comentan que:

Sé que vimos probabilidades. En inferencia vimos (...) cómo se llama eso (...) la t-Student y otras cosas. Pero si tuviera que decir que aprendí (...) no. Yo creo que didáctica de la estadística fue el curso que más me acercó a la estadística y probabilidad (...), por ejemplo, a la aleatoriedad que nunca en la vida se me hubiese ocurrido que tenía que estudiarlo en algún momento (Estudiante 5).

Faltó ver más contenidos, más lecturas, poner más en práctica la parte teórica más que solamente ejercicios, porque lo que veíamos estaba orientado más al cálculo que a la enseñanza (Estudiante 3).

Estos resultados de baja formación coinciden con lo mencionado en la literatura, que evidencian la escasa formación previa de los futuros profesores en las temáticas relacionadas con estadística descriptiva (ej., Batanero, Arteaga y Ruiz, 2010; Coutinho, Almouloud y Silva, 2012; Gea, Batanero, Fernándes, y Arteaga. 2016), que posiblemente aprendieron para aprobar la asignatura, es decir, tuvieron un aprendizaje superficial y no profundo. Esto implica, que los futuros profesores no están enfocados en el significado de lo que se desea enseñar, no relacionan lo que están aprendiendo con sus conocimientos previos y no tienden a integrar la teoría con la práctica (González, 2010), lo que repercutirá en su trayectoria profesional.

\section{Conclusiones}

Este estudio trató de analizar el efecto de la formación disciplinar en estadística, probabilidad y pedagógica recibida por un grupo estudiantes de Pedagogía en Educación Matemática de una universidad del centro-sur de Chile en los porcentajes de logro obtenidos en la END, atendiendo a la importancia que eventualmente tendrá esta evaluación en desempeño y carrera docente de los futuros profesores.

Los resultados obtenidos en la END por los estudiantes de Pedagogía en Educación matemática de una institución con una amplia trayectoria formativa, en las temáticas asociadas a estadística y probabilidad presentes en el currículo escolar se encuentran por debajo del nivel nacional. Ello evidencia que estos contenidos, siguen siendo un desafío en los itinerarios 
formativos para las instituciones formadoras, a pesar de haber pasado cerca de una década de su introducción en el currículo escolar chileno, tanto a nivel de enseñanza básica como media, situación que, a nivel internacional, fue analizada en los trabajos recogidos en Batanero, Burrill y Reading (2011)

Los programas de asignaturas de esta institución no se han renovado conforme a las nuevas normativas impuestas a nivel ministerial, ni tampoco se han alineado a los estándares orientadores para las carreras de pedagogía media publicados y socializados en las instituciones de educación superior a partir del año 2012 por el MINEDUC. Escenario, que devela una vez más que la formación disciplinar en estadística y probabilidad se lleva a cabo de manera fragmentada y procedimental lo que algunos autores han llamado conocimiento inerte (Bakker \& Derry, 2011).

La presente investigación muestra que existe una correlación estadísticamente significativa entre el resultado en las pruebas END y el rendimiento académico en las asignaturas de Inferencia Estadística, Currículo y Evaluación I, Currículo y Evaluación II, por tanto, dan indicios que una buena formación inicial del profesorado repercutirá en sus conocimientos futuros para llevar a cabo la enseñanza.

De lo contrario, la institución formadora seguirá enviado al mercado laboral profesionales con insuficientes conocimientos en temáticas asociadas a estadística, probabilidad y su evaluación, tópicos que permitirían alfabetizar estadísticamente y probabilística (Gal, 2002; 2004; 2019) de manera de formar ciudadanos críticos en la actual era de la información y la tecnología. De otro modo, los estudiantes en su paso por la educación obligatoria, aprenderán una gran cantidad de contenidos, conceptos y procedimientos estadísticos que no serán capaces de aplicar cuando enfrenten problemas reales (Bakker, Van Mierlo, \& Akkerman, 2012; ZapataCardona, 2016).

Por otro lado, de las entrevistas a los futuros profesores, se desprende los tópicos relacionados con estadística descriptiva debe ser reestructurados en los programas de asignatura, explicitando claramente su aporte a la alfabetización estadística y su alineación con los contenidos que deberán tratar a nivel de sistema escolar. Además, se debe orientar sus prácticas evaluativas acorde a los mencionado en el informe GAISE (Guidelines for Assessment and Instruction in Statistics Education; Franklin et al. 2007), es decir, abordar las temáticas afines a estadística y probabilidad desde una nueva mirada y no seguir reproduciendo la enseñanza de 
estos tópicos desde la mirada matemática, como se ha estado haciendo hasta el momento. Asimismo, potenciar la asignatura de didáctica de la estadística la cual genera un puente entre lo pedagógico y disciplinar en estas temáticas.

Finalmente, el presente estudio proporciona evidencias empíricas sobre la incidencia de la formación en estadística, probabilidad y evaluación en los resultados de la END. Antecedentes útiles para las instituciones de educación superior que poseen en su oferta académica la carrera de pedagógica en matemática con características similares, para dar respuesta a los desafíos que plantea la Ley 20.903 de manera de orientar procesos de monitoreo y la implementación de planes remediales que deben formular las instituciones formadoras de manera de potenciar la formación inicial, acciones que han sido demandas por décadas en las investigaciones afines a la Formación Inicial Docente.

\section{Agradecimientos}

Investigación Financiada por Dirección de Investigación Universidad del Bio-Bío (DIUBB). Código DICA 195723 4/IenDU-FID. "La enseñanza y la evaluación en estadística y probabilidad: Una mirada desde los profesores en activo y en formación”.

\section{Referencias}

Arteaga, P., Batanero, C., Cañadas, G., \& Contreras, J. M. (2011). Las tablas y gráficas como objetos culturales. Números, 76, 55-67

Ávalos, B. (2008). Formación inicial de profesores. Formación inicial docente en Chile: calidad y políticas. En C. Bellei, D. Contreras \& J. Valenzuela (Eds.), Ecos de la Revolución Pingüino: avances, debates y silencios de la reforma educacional. Santiago: Universidad de Chile.

Batanero, C., Arteaga, P., \& Ruiz, B. (2010). Análisis de la complejidad semiótica de los gráficos producidos por futuros profesores de educación primaria en una tarea de comparación de dos variables estadísticas. Enseñanza de las Ciencias, 28(1), 141-154.

Batanero, C., Burrill, G., \& Reading, C. (Eds.). (2011). Teaching statistics in school mathematics-challenges for teaching and teacher education: New York; Springer.

Ball, D.L., Thames, M.H., \& Phelps, G. (2008). Content knowledge for teaching: What makes it special? Journal of Teacher Education, 59(5), 389-407.

Bakker, A. \& Derry, J. (2011). Lessons from inferentialism for statistics education. Mathematical Thinking and Learning, 13(1-2), 5-26. https://doi.org/10.1080/10986065.2011.538293

Bakker, A., Van Mierlo, X., \& Akkerman, S. (2012, junio). Learning to integrate statistical and 
work related reasoning. Trabajo presentado en el 12th International Congress on Mathematical Education. Seoul: ICMI.

Ben-Zvi, D. \& Garfield, J. (2004). Statistical literacy, reasoning, and thinking: Goals, definitions, and challenges. En D. Ben-Zvi \& J. Garfield (Eds.), The challenge of developing statistical literacy, reasoning and thinking (pp. 3-15). Dordrecht, The Netherlands: Kluwer.

Cornejo, J., Sanhueza, S., \& Rioseco, M. (2012). Orientaciones para la elaboración de tesis, seminarios y paper académicos. Talca: Universidad Católica del Maule.

Cobb, G. \& Moore, D. (1997). Mathematics, statistics, and teaching. American Mathematical Monthly, 104(9), 801-823. https://doi.org/10.1080/00029890.1997.11990723

Coutinho, C., Almouloud, S., \& Silva, M. J. (2012). ). O desenvolvimento do letramento estatístico a partir do uso do Geogebra: Um estudo com professores de matemática. REVEMAT, 2(7), 246-265. http://dx.doi.org/10.5007/1981- 1322.2012v7n2p246.

Engel, J. (2019). Cultura estadística y sociedad. En J. M. Contreras, M. M. Gea, M. M. LópezMartín \& E. Molina-Portillo (Eds.), Actas del Tercer Congreso Internacional Virtual de Educación Estadística (pp. 1-18). Granada: Grupo FQM-126. Disponible en www.ugr.es/local/fqm126/civeest.html

Estrella, S., Olfos, R., \& Mena-Lorca, A. (2015). Pedagogical content knowledge of statistics among primary school teachers. Educação e Pesquisa, 41(2), 477-493. https://doi.org/10.1590/s1517-97022015041858

Fernandes, J. A., Gea, M. M., \& Correia, P. F. (2019). Conocimiento de la estadística bivariada de los futuros profesores de portugués desde los primeros años. Revista Portuguesa de Educação, 32(2), 40-56. https://dx.doi.org/10.21814/rpe.16121.

Feiman-Nemser, S., \& Buchman, M. (1988). Lagunas en las prácticas de enseñanza de los programas de formación del profesorado. En L. M. Villar (Ed.), Conocimientos, creencias y teorías de los profesores (pp. 301-314). Alcoy: Marfil.

Franklin, C., Kader. G., Mewborn, D., Moreno, J., Peck, R., Perry, M., \& Scheaffer, R. (2007). Guidelines for assessment and instruction in statistics education (GAISE) report: A Pre-K12 curriculum framework. Alexandria, VA: American Statistical Association. Disponible en: www.amstat.org/Education/gaise/..

Gal, I. (2002). Adults' statistical literacy: Meaning, components, responsibilities, International Statistical Review, 70(1), 1-25.

Gal, I. (2004). Statistical literacy meanings, components, responsibilities. En D. Ben-Zvi \& J. Garfield (Eds.), The challenge of developing statistical literacy, reasoning and thinking (pp. 47-78). Netherlands: Springer. https://doi.org/10.1007/1-4020-2278-6_3.

Gal, I. (2019). Understanding statistical literacy: About knowledge of contexts and models. En J. M. Contreras, M. M. Gea, M. M. López-Martín \& E. Molina-Portillo (Eds.), Actas del Tercer Congreso Internacional Virtual de Educación Estadística (pp. 1-15). Granada: Grupo FQM-126. Disponible en www.ugr.es/local/fqm126/civeest.html

Gea, M.M., Arteaga, P., \& Cañadas, G. (2017). Interpretación de gráficos estadísticos por futuros profesores de educación secundaria. Avances de Investigación en Educación Matemática, 12, 19-37. https://doi.org/10.35763/aiem.v1i12.189. 
Gea. M.M., Batanero, C., Arteaga, P., \& Estepa, A. (2019). Conocimiento especializado de correlación y regresión en futuros profesores de educación secundaria. Profesorado, 23(2), 397-419. https://doi.org/10.30827/profesorado.v23i2.9693.

Gea, M. M., Batanero, C., Fernándes, J. A., \& Arteaga, P. (2016). Interpretación de resúmenes estadísticos por futuros profesores de educación secundaria. REDIMAT, 5(2), 135-157.

Gómez, E., Batanero, C., \& Contreras, J. M. (2014). Conocimiento matemático de futuros profesores para la enseñanza de la probabilidad desde el enfoque frecuencial. BOLEMA. Boletim de Educação Matemática, 28(48), 209-229.

González, C. (2010). El aprendizaje y el conocimiento académico sobre la enseñanza como claves para mejorar la docencia universitaria. Calidad en la Educación, 33, 123-146. https://dx.doi.org/10.31619/caledu.n33.141

Hill, H. C., Ball, D. L., \& Schilling, S. G. (2008). Unpacking pedagogical content knowledge: Conceptualizing and measuring teachers' topic-specific knowledge of students. Journal for Research in Mathematics Education, 39(4), 372-400.

Kvale, S. (2011). Las entrevistas en investigación cualitativa. Madrid: Morata.

McMillan, J., \& Schumacher, S. (2011). Investigación educativa. Madrid: Pearson.

Marcelo, C. (2009). El profesorado principiante: Inserción a la docencia. Barcelona: Octaedro.

Marcelo, C. \& Vaillant, D. (2018a). La formación inicial docente: problemas complejosrespuestas disruptivas. Cuadernos de Pedagogía, 489, 29-32.

Marcelo, C. \& Vaillant, D. (2018b). Hacia una formación disruptiva de docentes. 10 claves para el cambio. Madrid: Narcea.

Mayring, P. (2000) Qualitative content analysis. Forum qualitative social research, 1(2), 1-10 https://dx.doi.org/10.17169/fqs-1.2.1089

MINEDUC. (2009). Objetivos fundamentales y contenidos mínimos obligatorios de la Educación Básica y Media. Santiago: Ministerio de Educación.

MINEDUC. (2012). Estándares orientadores para egresados de pedagogía en educación media. Santiago: LOM Ediciones Ltda.

MINEDUC (2016). Sistema de desarrollo profesional docente (Ley 20.903). Valparaíso: Congreso Nacional de Chile.

Ponte, J. P. \& Chapman, O. (2006). Mathematics teachers' knowledge and practices. En A. Gutiérrez y P. Boero (Eds.), Handbook of research on the psychology of mathematics education: Past, present and future (pp. 461-494). Roterdham: Sense.

Rodríguez-Alveal, F., Díaz-Levicoy, D., \& Maldonado-Fuentes, A. C. (2018). Evaluación del conocimiento y argumentación adquiridos por futuros profesores de secundaria de matemática sobre índices de resumen numérico. Investigación y Postgrado, 33(2), 97-114.

Rodríguez-Alveal, F., Díaz-Levicoy, D., \& Vásquez, C. (2018). Evaluación de la alfabetización probabilística del profesorado en formación y en activo. Estudios Pedagógicos, 44(1), 135156. https://dx.doi.org/10.4067/S0718-07052018000100135.

Rodríguez-Alveal, F., Maldonado-Fuentes, A. C., \& Sandoval, P. (2016). Comprensión de las 
medidas de tendencia central: un estudio comparativo en estudiantes de pedagogía en matemática en dos instituciones formadoras chilenas. Avaliação, 21(3), 929-952. https://dx.doi.org/10.1590/S1414-40772016000300013

Rodríguez-Alveal, F. \& Sandoval, P. (2012). Habilidades de codificación y descodificación de tablas y gráficos estadísticos: un estudio comparativo en profesores y alumnos de pedagogía en enseñanza básica. Avaliação, 17(1), 207-235. https://dx.doi.org/10.1590/S1414$\underline{40772012000100011}$

Shulman, L.S. (1986). Those who understand. Knowledge growth in teaching. Educational Researcher, 15(2), 4-14.

Vaillant, D. (2007). Mejorando la formación y el desarrollo profesional docente en Latinoamérica. Pensamiento Educativo, 41(2), 207-222.

Vaillant, D., \& Marcelo, C. (2001). Tareas del formador. Archidona: Aljibe.

Vezub, L.F. (2016). Los saberes docentes en la formación inicial. La perspectiva de los formadores. Pensamiento Educativo, 53(1), 1-14. https://dx.doi.org/10.7764/PEL.53.1.2016.9.

Zapata-Cardona, L. (2016). Enseñanza de la estadística desde una perspectiva crítica. Yupana, 10, 30-41. https://doi.org/10.14409/yu.v0i10.7695.

Autores

Francisco Rodríguez-Alveal

Profesor de Estado en Matemática (UBB). Magíster en Bioestadística (UCHILE). Doctor en Educación (UBB). Académico Departamento Ciencias de la Educación, Facultad de Educación y Humanidades, Universidad del Bío-Bío (UBB). Línea de investigación: Formación Inicial Docente y Didáctica de la Estadística. E-mail: frodriguez@ubiobio.cl

Danilo Díaz-Levicoy

Profesor de Matemática y Computación (ULAGOS). Máster en Didáctica de la Matemática (UGR).

Doctor en Ciencias de la Educación (UGR). Académico Departamento de Matemática, Física y Estadística, Facultad de Ciencias Básicas, Universidad Católica del Maule. Miembro del Centro de Investigación en Educación Matemática y Estadística, Universidad Católica del Maule (UCM). Línea de Investigación: Didáctica de la Matemática y la Estadística. Email: dddiaz01@hotmail.com 